\title{
The Safety and Clinical Outcomes of Chemoembolization in Child-Pugh Class C Patients with Hepatocellular Carcinomas
}

Tae Won Choi, $M D^{1}$, Hyo-Cheol Kim, MD ${ }^{1}$, Jeong-Hoon Lee, $M D^{2}$, Su Jong $Y u, M D^{2}$, Beomsik Kang, $M D^{1}$, Saebeom Hur, $M D^{1}$, Myungsu Lee, $M D^{1}$, Hwan Jun Jae, $M D^{1}$, Jin Wook Chung, MD ${ }^{1}$

Departments of ${ }^{1}$ Radiology and ${ }^{2}$ Internal Medicine, Seoul National University College of Medicine, Seoul National University Hospital, Seoul 03080, Korea

Objective: To evaluate the safety and clinical outcomes of chemoembolization in Child-Pugh class C patients with hepatocellular carcinomas (HCC).

Materials and Methods: The study comprised 55 patients with HCC who were classified as Child-Pugh class C and who underwent initial chemoembolization between January 2003 and December 2012. Selective chemoembolization was performed in all technically feasible cases to minimize procedure-related complications. All adverse events within 30 days were recorded using the Common Terminology Criteria for Adverse Events (CTCAE). The tumor response to chemoembolization was evaluated using the modified Response Evaluation Criteria In Solid Tumors.

Results: Thirty (54.5\%) patients were within the Milan criteria, and $25(45.5 \%)$ were beyond. The mortality of study subjects at 30 days was $5.5 \%$. Major complications were observed in five $(9.1 \%)$ patients who were all beyond the Milan criteria: two hepatic failures, one hepatic encephalopathy, and two CTCAE grade 3 increases in aspartate aminotransferase/ alanine aminotransferase abnormality. The mean length of hospitalization was $6.3 \pm 8.3$ days (standard deviation), and 18 $(32.7 \%)$ patients were discharged on the next day after chemoembolization. The tumor responses of the patients who met the Milan criteria were significantly higher $(p=0.014)$ than those of the patients who did not. The overall median survival was 7.1 months ( $95 \%$ confidence interval: 4.4-9.8 months).

Conclusion: Even in patients with Child-Pugh class C, chemoembolization can be performed safely with a selective technique in selected cases with a small tumor burden.

Index terms: Hepatocellular carcinoma; Chemoembolization; Liver cirrhosis; Liver failure; Safety

Received April 27, 2015; accepted after revision July 11, 2015. This study was supported by the National Research Foundation of Korea (NRF) funded by the Korean government (NRF-

2013R1A1A2A10011007).

Corresponding author: Hyo-Cheol Kim, MD, Department of Radiology, Seoul National University College of Medicine, Seoul National University Hospital, 101 Daehak-ro, Jongno-gu, Seoul 03080, Korea.

- Tel: (822) 2072-2584 - Fax: (822) 743-6385

- E-mail: angiointervention@gmail.com

This is an 0pen Access article distributed under the terms of the Creative Commons Attribution Non-Commercial License (http://creativecommons.org/licenses/by-nc/3.0) which permits unrestricted non-commercial use, distribution, and reproduction in any medium, provided the original work is properly cited.

\section{INTRODUCTION}

Hepatocellular carcinoma (HCC) patients with Child-Pugh class $\mathrm{C}$ disease are treated with supportive care according to the Barcelona clinic liver cancer (BCLC) staging system (1) or by liver transplantation in cases of limited tumor burden (2). Given the shortage of donors, liver transplantation cannot be performed in all patients with Child-Pugh class $C$ disease. Although chemoembolization has a survival benefit over conservative management $(3,4)$, Child-Pugh class $C$ is one of the contraindications for chemoembolization (5). According to the guidelines of the Japan Society of 
Hepatology, subsegmental chemoembolization can be performed as a compassionate treatment in Child-Pugh class $C$ cases when hepatic encephalopathy and intractable ascites are absent and the serum bilirubin level is less than $3 \mathrm{mg} / \mathrm{dL}(6)$.

With advancements in microcatheter technology, selective chemoembolization has been adapted by many interventional radiologists, resulting in excellent clinical outcomes and fewer complications $(7,8)$. In actual clinical practice, chemoembolization can occasionally be considered even in patients with Child-Pugh class $\mathrm{C}$ according to the patient's individual clinical situation, including the tumor burden, need for local control, and availability of liver transplantation. However, to the best of our knowledge, there has been no report on the safety and clinical outcomes of chemoembolization in patients with decreased liver function classified as Child-Pugh class C. The aim of this study was to evaluate the safety and clinical outcomes of chemoembolization in Child-Pugh class C patients with HCC.

\section{MATERIALS AND METHODS}

\section{Patients}

This study was approved by the Institutional Review Board of our institute, and the requirement for informed patient consent was waived because of its retrospective design.

From January 2003 to December 2012, 5264 HCC patients received initial chemoembolization at our institute according to the chemoembolization database. The inclusion criteria were as follows: 1) patients with underlying liver cirrhosis and decreased hepatic function categorized as Child-Pugh class $C$; 2) HCC diagnosed either by pathology or by non-invasive imaging modalities according to American Association for the Study of Liver Diseases practice guidelines (9); and 3) no medical history of previous chemoembolization. The exclusion criteria were as follows: 1) previous therapy, such as percutaneous alcohol injection, radiofrequency ablation, or surgical resection; 2) patients with ruptured $\mathrm{HCC} ; 3$ ) concomitant malignant tumors in addition to $\mathrm{HCC}$; or 4 ) an aborted chemoembolization procedure caused by a severe arterioportal shunt. Fifty-five patients (46 men and 9 women; mean age, 54 years; range, 34-77 years) with Child-Pugh class $C$ were included in this retrospective study.

Liver transplantation was recommended as the first treatment in all patients with Child-Pugh class $\mathrm{C}$ disease and a limited tumor burden within the Milan criteria (10). If liver transplantation was not feasible, the treatment strategy (chemoembolization and supportive care) was determined for each patient based on the attending physician's recommendations and the patient's choice. Patients made their choice based on the physician's advice that chemoembolization could delay tumor progression but that it could also increase the risk of hepatic failure. Patients who feared hepatic failure received supportive care.

\section{Chemoembolization}

All patients underwent contrast-enhanced computed tomography (CT) and/or magnetic resonance imaging (MRI) within 40 days before the chemoembolization procedure. The methods and techniques of chemoembolization at our institution are summarized as follows: First, arteriography of the celiac and superior mesenteric arteries was performed with a 5-Fr angiographic catheter (RH catheter; Cook, Bloomington, IN, USA) to evaluate the anatomical variation of the hepatic arteries, the location and extent of the HCC, and the tumor-feeding arteries. Selective chemoembolization of the subsegmental hepatic artery was initially considered and performed in all technically feasible cases to minimize procedure-related complications such as hepatic function deterioration. After the microcatheter with a 2.0-Fr tip (Progreat; Terumo, Tokyo, Japan) or a 2.4-Fr tip (Microferret-18; Cook) was advanced into the most distal branches of the tumor-feeding artery that were technically accessible, an emulsion of iodized oil (Lipiodol; Laboratoire Andre Guerbet, Aulnay-sous-Bois, France) mixed with doxorubicin hydrochloride (Adriamycin RDF; Ildong, Seoul, Korea) was infused via the microcatheter until a decrease in the blood flow to the tumor was observed (Fig. 1). The mean dose of doxorubicin hydrochloride was 26.0 $\pm 12.1 \mathrm{mg}$, and the median dose was $30 \mathrm{mg}$. Additional embolization was performed with $1 \mathrm{~mm}$-sized absorbable gelatin sponge particles (Gelform; Upjohn, Kalamanzoo, MI, USA or Cutanplast; Mascia Brunelli, Milan, Italy) to maximize the therapeutic effect of the chemoembolization.

Follow-up contrast-enhanced CT or MRI examinations were performed at intervals of two to three months thereafter. When a recurrent or residual tumor was identified on CT or MRI, chemoembolization was repeated in the same manner. All patients regularly visited the outpatient clinic of the attending physician (hepatologist) at intervals of two to four weeks, and treatment triage was performed by the attending physician. 


\section{Baseline Data Collection}

The patients' medical records were reviewed, and the following clinical information and laboratory parameters before chemoembolization procedure were obtained by two authors: age, sex; etiology of HCC; Eastern Cooperative Oncology Group (ECOG) performance status; Child-Pugh score; Model for End-Stage Liver Disease (MELD) score; and laboratory data, including albumin, bilirubin, the international normalized ratio (INR), creatinine, alphafetoprotein (AFP), aspartate aminotransferase (AST), and alanine aminotransferase (ALT). All baseline laboratory parameters were recorded from the tests that were performed closest to the chemoembolization procedure and graded using National Cancer Institute Common Terminology Criteria for Adverse Events (CTCAE) version 4.0 (11).

The patients' American Joint Committee on Cancer (AJCC)/ Union for International Cancer Control (UICC) staging, Okuda staging, and Cancer of the Liver Italian Program (CLIP) scores were also determined from the clinical and laboratory data and from analysis of the pre-procedural contrastenhanced CT or MRI. The two radiologists analyzed the CT or MRI images and reached a consensus about the extent of the tumor and the presence of portal vein thrombosis (PVT), which is required to determine AJCC staging, Okuda staging, and CLIP score. In addition, two reviewers determined whether a given patient's tumor burden was within the Milan criteria.

\section{Safety}

The patients' follow-up medical records after the chemoembolization procedure were reviewed, and their laboratory data were analyzed by two authors. All adverse events and mortalities that occurred within one month after the chemoembolization were recorded. However, clinical symptoms and signs of postembolization syndrome were not analyzed in this study because of its subjective nature

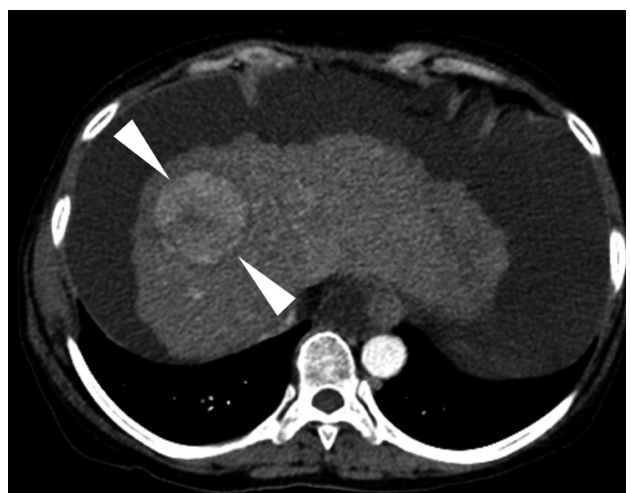

A

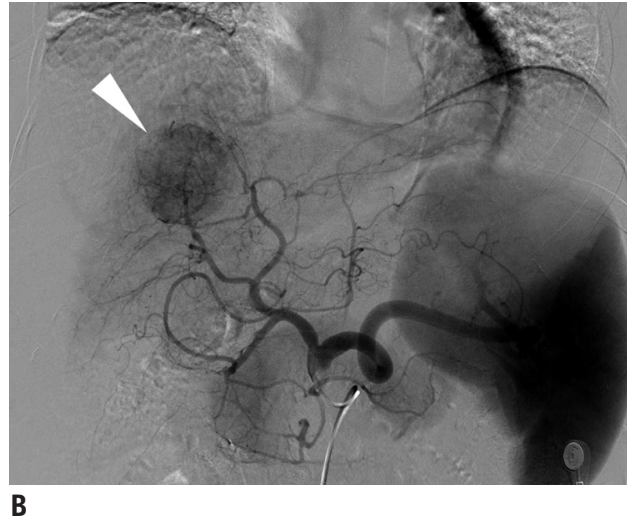

B

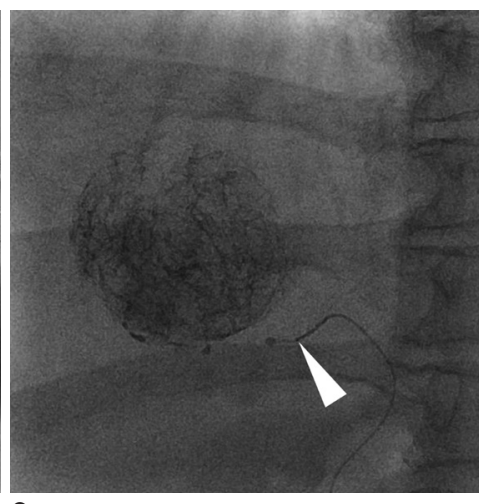

C

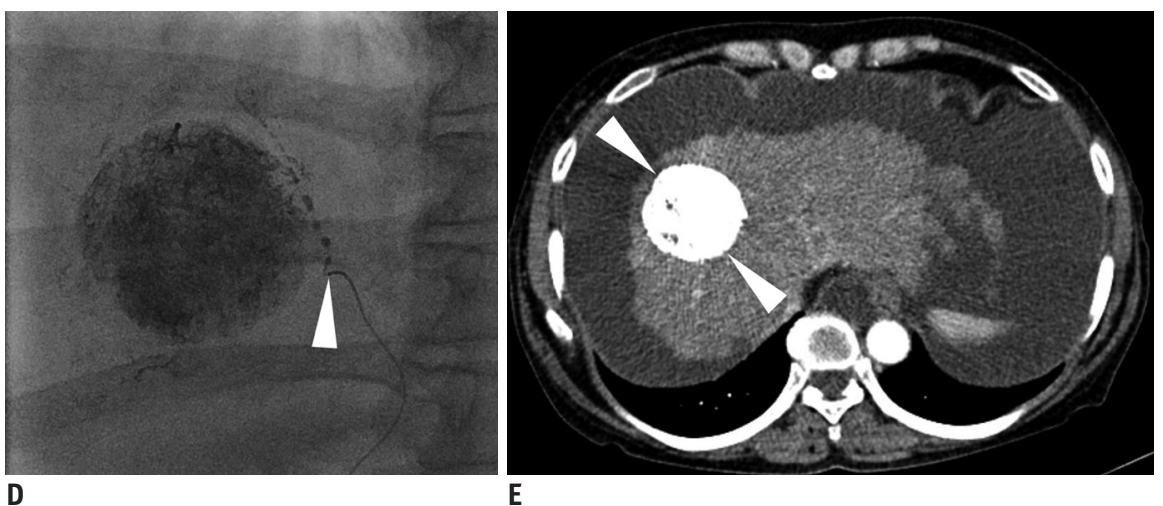

Fig. 1. 52-year-old woman with Child-Pugh class $\mathrm{C}$ liver cirrhosis.

A. Axial CT image obtained at arterial phase shows $4.5 \mathrm{~cm}$ arterial enhancing mass (arrowheads) in segment 4 of liver. Note cirrhotic liver and large amount of ascites. B. Celiac arteriography shows hypervascular tumor staining (arrowhead) that is supplied by two prominent feeding arteries from left hepatic artery. C. Tip of microcatheter (arrowhead) was placed at distal portion of one of tumor-feeding arteries and followed by infusion of iodized oil emulsion. D. Thereafter, other tumor-feeding branch from left hepatic artery was selected and catheterized with microcatheter (arrowhead), and chemoembolization was performed. Spot image obtained during chemoembolization shows additional dense accumulation of iodized oil in tumor and oily portogram around tumor. E. Arterial phase image of follow-up liver CT scan shows dense accumulation of iodized oil in previously noted hepatocellular carcinoma in segment 4 (arrowheads) with no evidence of viable tumor. 
and self-limited clinical course (12). Abnormal laboratory test results after the chemoembolization were recorded and graded according to the CTCAE. Because laboratory test results are commonly abnormal in Child-Pugh class $C$ patients, the severity of chemoembolization-related adverse effects on laboratory parameters was evaluated by comparing the CTCAE grade of the laboratory abnormalities after chemoembolization with the grade at baseline.

To evaluate and determine risk factors for chemoembolization-related complications, demographic data including age and sex, performance status, Child-Pugh score, tumor staging (AJCC/UICC staging, Okuda staging, CLIP score and MELD score), presence of PVT and laboratory parameters (albumin, bilirubin, and the INR) of the patients who developed major complications were compared with those of the remaining patients.

In addition, the duration of hospitalization for all patients after chemoembolization was recorded, and the cause of extended hospitalization was assessed for patients who were hospitalized for more than seven days after the procedure.

\section{Treatment Response}

In patients who underwent follow-up contrast-enhanced CT or MRI after chemoembolization, two radiologists evaluated the tumor response to chemoembolization and reached a consensus using the modified Response Evaluation Criteria In Solid Tumors (13).

\section{Survival}

Survival analysis was performed, and survival curves were calculated from the time of the chemoembolization procedure in all patients. Patient follow-up ended when any of the following criteria was met: 1) death of the patient, 2) liver transplantation, or 3) the end of the study, which was 31 August 2014. If a patient underwent transplantation, the follow-up data were censored on the date of the operation. The survival information, including the date of the patient's death and liver transplantation, was obtained by reviewing our institution's medical records. In addition, we contacted the Resident Service Division of the Ministry of Public Administration and Security for survival information on the patients who were lost to follow-up at our institution.

\section{Statistical Analysis}

Differences between the patients who developed major complications and those who did not were evaluated by a Fisher's exact test, a Student's $t$ test, or a Mann-Whitney U test. In addition, variables of the patients who developed major or minor complications were compared with those who had no adverse events using identical statistical methods. The overall median survival times and survival curves were calculated with the Kaplan-Meier method, and subgroup comparisons were performed using a logrank test. In addition, a Cox proportional hazards model was used for multivariate analysis. The tumor response to chemoembolization between subgroups was compared using a Mann-Whitney U test. All statistical analyses were performed with SPSS version 19.0 software (SPSS Inc., Chicago, IL, USA), and a $p$ value less than 0.05 was considered statistically significant in all of the analyses.

\section{RESULTS}

\section{Patients and Baseline Characteristics}

The demographic data and baseline laboratory parameters of the 55 patients are summarized in Table 1. Thirty (54.5\%) patients were within the Milan criteria, and 25 (45.5\%) were beyond them.

\section{Morbidity and Mortality}

Mortality at 30 days was 5.5\% (3/55), two deaths from acute hepatic failure and one from disease progression. of these three patients, two expired at the hospital.

Major complications were observed in five $(9.1 \%)$ patients. Two of these developed hepatic failure and expired, one patient developed hepatic encephalopathy, and two patients had a CTCAE grade 3 increase in AST/ALT abnormalities. Twenty-eight (50.9\%) patients showed only a CTCAE grade 1 or 2 increase in laboratory test parameters, and there were no complications in the remaining 22 $(40.0 \%)$ patients. The gradients in the CTCAE grade in the laboratory parameters from baseline of all patients after the chemoembolization procedure are presented in Table 2.

All of the patients $(5 / 5)$ who developed major complications after chemoembolization had tumor burden beyond the Milan criteria, whereas only $40.0 \%(20 / 50)$ of those without complications or with minor complications had tumor burden beyond the Milan criteria $(p=0.015)$. In addition, the patients with major complications had significantly higher T-staging $(p=0.031)$ and higher CLIP scores $(p=0.007)$ compared with those without complications or with minor complications. However, the characteristics of the patients who developed major or minor complications were not significantly different from 
Table 1. Baseline Patient Characteristics

\begin{tabular}{|c|c|c|c|c|}
\hline Characteristics & Total & $\begin{array}{l}\text { Patients with Major } \\
\text { Complications }\end{array}$ & $\begin{array}{c}\text { Patients with } \\
\text { Minor Complication } \\
\text { or without Complication }\end{array}$ & $P$ \\
\hline Number & 55 & 5 & 50 & \\
\hline Age, mean (range) & $54(34-77)$ & $52(41-64)$ & $55(34-77)$ & 0.631 \\
\hline Sex (M:F) & $46: 9$ & $4: 1$ & 42:8 & \\
\hline \multicolumn{5}{|l|}{ Etiology } \\
\hline HBV & $38(69.1 \%)$ & $4(80.0 \%)$ & $34(68.0 \%)$ & \\
\hline $\mathrm{HCV}$ & $5(9.1 \%)$ & $0(0.0 \%)$ & $5(10.0 \%)$ & \\
\hline Alcohol & $7(12.7 \%)$ & $0(0.0 \%)$ & $7(14.0 \%)$ & \\
\hline Others & $5(9.1 \%)$ & $1(20.0 \%)$ & $4(8.0 \%)$ & \\
\hline ECOG performance status & & & & 0.251 \\
\hline 0 & $0(0.0 \%)$ & $0(0.0 \%)$ & $0(0.0 \%)$ & \\
\hline 1 & $24(43.6 \%)$ & $2(40.0 \%)$ & $22(44.0 \%)$ & \\
\hline 2 & $28(50.9 \%)$ & $2(40.0 \%)$ & $26(52.0 \%)$ & \\
\hline 3 or more & $3(5.5 \%)$ & $1(20.0 \%)$ & $2(4.0 \%)$ & \\
\hline Child-Pugh score & & & & 0.496 \\
\hline 10 & $37(67.3 \%)$ & $4(80.0 \%)$ & $33(66.0 \%)$ & \\
\hline 11 & $16(29.1 \%)$ & $1(20.0 \%)$ & $15(30.0 \%)$ & \\
\hline 12 & $2(3.6 \%)$ & $0(0.0 \%)$ & $2(4.0 \%)$ & \\
\hline MELD score, mean $\pm S D$ & $18.09 \pm 2.81$ & $16.40 \pm 1.95$ & $18.22 \pm 2.84$ & 0.169 \\
\hline Clinical AJCC/UICC T staging & & & & 0.031 \\
\hline $\mathrm{T} 1$ & $23(41.8 \%)$ & $0(0.0 \%)$ & $23(46.0 \%)$ & \\
\hline T2 & $16(29.1 \%)$ & $2(40.0 \%)$ & $14(28.0 \%)$ & \\
\hline Т3а & $3(5.5 \%)$ & $0(0.0 \%)$ & $3(6.0 \%)$ & \\
\hline T3b & $12(21.8 \%)$ & $3(60.0 \%)$ & $9(18.0 \%)$ & \\
\hline $\mathrm{T} 4$ & $1(1.8 \%)$ & $0(0.0 \%)$ & $1(2.0 \%)$ & \\
\hline CLIP score & & & & 0.007 \\
\hline 1 & $0(0.0 \%)$ & $0(0.0 \%)$ & $0(0.0 \%)$ & \\
\hline 2 & $20(36.4 \%)$ & $0(0.0 \%)$ & $20(40.0 \%)$ & \\
\hline 3 & $17(30.9 \%)$ & $1(20.0 \%)$ & $16(32.0 \%)$ & \\
\hline 4 & $8(14.5 \%)$ & $1(20.0 \%)$ & $7(14.0 \%)$ & \\
\hline 5 & $7(12.7 \%)$ & $1(20.0 \%)$ & $6(12.0 \%)$ & \\
\hline 6 & $3(5.5 \%)$ & $2(40.0 \%)$ & $1(2.0 \%)$ & \\
\hline Okuda staging & & & & 0.156 \\
\hline 1 & $0(0.0 \%)$ & $0(0.0 \%)$ & $0(0.0 \%)$ & \\
\hline 2 & $31(56.4 \%)$ & $1(20.0 \%)$ & $30(60.0 \%)$ & \\
\hline 3 & $24(43.6 \%)$ & $4(80.0 \%)$ & $20(40.0 \%)$ & \\
\hline Milan criteria & & & & 0.015 \\
\hline In & $30(54.5 \%)$ & $0(0.0 \%)$ & $30(60.0 \%)$ & \\
\hline Out & $25(45.5 \%)$ & $5(100.0 \%)$ & $20(40.0 \%)$ & \\
\hline Portal vein thrombosis & & & & 0.080 \\
\hline Absent & $42(76.4 \%)$ & $2(40.0 \%)$ & $40(80.0 \%)$ & \\
\hline Present & $13(23.6 \%)$ & $3(60.0 \%)$ & $10(20.0 \%)$ & \\
\hline Albumin, mean $\pm S D(g / d L)$ & $2.49 \pm 0.26$ & $2.46 \pm 0.23$ & $2.49 \pm 0.27$ & 0.797 \\
\hline Bilirubin, mean $\pm \mathrm{SD}(\mathrm{mg} / \mathrm{dL})$ & $4.65 \pm 3.62$ & $5.12 \pm 1.45$ & $4.60 \pm 3.77$ & 0.764 \\
\hline INR, mean \pm SD & $1.72 \pm 0.29$ & $1.43 \pm 0.19$ & $1.75 \pm 0.28$ & 0.015 \\
\hline Creatinine, mean $\pm \mathrm{SD}(\mathrm{mg} / \mathrm{dL})$ & $0.91 \pm 0.22$ & $0.81 \pm 0.03$ & $0.92 \pm 0.23$ & 0.002 \\
\hline $\mathrm{AST}$, mean $\pm \mathrm{SD}(\mathrm{IU} / \mathrm{L})$ & $100.4 \pm 68.4$ & $141.4 \pm 85.6$ & $96.3 \pm 66.1$ & 0.161 \\
\hline ALT, mean $\pm S D(I U / L)$ & $52.9 \pm 31.7$ & $60.0 \pm 30.7$ & $52.1 \pm 32.0$ & 0.602 \\
\hline
\end{tabular}

AJCC/UICC = American Joint Committee on Cancer/Union for International Cancer Control, ALT = alanine aminotransferase, AST $=$ aspartate aminotransferase, CLIP $=$ Cancer of the Liver Italian Program, ECOG = Eastern Cooperative Oncology Group, INR $=$ international normalized ratio, MELD = Model for End-Stage Liver Disease, $S D=$ standard deviation 
those of the patients who had no adverse events.

\section{Duration of Hospitalization}

The mean length of hospitalization was $6.3 \pm 8.3$ days (standard deviation), and the median length was three days (range 1-41 days). Eighteen (32.7\%) patients were discharged on the next day after chemoembolization without complications. Thirteen (23.6\%) patients were hospitalized for more than seven days after chemoembolization. The causes of extended hospitalization were as follows: one patient developed hepatic failure and expired; one developed hepatic encephalopathy; one was treated for obstructive jaundice caused by bile duct invasion of the tumor; three received management for preexisting intractable ascites or pleural effusions; four received supportive care for postembolization syndrome; one was treated for septic arthritis; one received conservative management for neutropenia of unknown cause; and one underwent a transplantation work-up.

\section{Tumor Response}

Follow-up contrast-enhanced CT or MRI was obtained in 43 patients, and the time interval between chemoembolization and CT/MRI ranged from 17 to 110 days (mean, 69 days; median 77 days). Of these 43 patients, $19(44.2 \%)$ showed complete responses to the chemoembolization, $10(23.3 \%)$ showed partial responses,
$9(20.1 \%)$ remained stable, and $5(11.6 \%)$ progressed. The tumor responses of the patients who met the Milan criteria were significantly higher $(p=0.014)$ than those of the patients who did not. Complete responses were achieved in $57.1 \%(16 / 28)$ of patients in whom the tumor burden was within the Milan criteria (Table 3 ).

\section{Survival}

At the end of the study, which was 31 August 2014, $22 \%(11 / 55)$ of all patients were alive. The overall median survival of all patients after chemoembolization was 7.1 months (95\% confidence interval: 4.4-9.8 months). Table 4 displays the results of the univariate analysis, which showed that the following baseline characteristics were associated with significantly higher survival rates: ECOG performance status of 1 or less, AJCC T stage 1, CLIP score of 3 or less, tumor burden within the Milan criteria, absence of PVT, low serum AFP ( $\leq 400 \mathrm{ng} / \mathrm{mL})$ and AST ( $\leq 80 \mathrm{IU} / \mathrm{L})$ levels, and the presence of objective tumor response (complete or partial response) (Fig. 2). In addition, the multivariate analysis identified four independent predictive factors for a shorter survival time: ECOG performance status $>1$, tumor burden that exceeded the Milan criteria, serum AST $>80$ $\mathrm{IU} / \mathrm{L}$, and the absence of objective tumor response (Table 5).

\section{Liver Transplantation}

The follow-up data revealed that seven (12.7\%) of 55

Table 2. Adverse Effects of Chemoembolization on Laboratory Parameters

\begin{tabular}{|c|c|c|c|c|c|}
\hline \multirow{3}{*}{ Characteristics } & \multicolumn{5}{|c|}{ No. Patients (\%) } \\
\hline & \multirow{2}{*}{ Total } & \multicolumn{4}{|c|}{ Increase in CTCAE (Version 4.0) Grade } \\
\hline & & 1 & 2 & 3 & 4 \\
\hline Decrease of serum albumin & $1(1.8 \%)$ & $1(1.8 \%)$ & 0 & 0 & 0 \\
\hline Elevation of total bilirubin & $11(20.0 \%)$ & $11(20.0 \%)$ & 0 & 0 & 0 \\
\hline INR prolongation & $9(16.4 \%)$ & $9(16.4 \%)$ & 0 & 0 & 0 \\
\hline Elevation of serum creatinine & $1(1.8 \%)$ & $1(1.8 \%)$ & 0 & 0 & 0 \\
\hline Elevation of AST and/or ALT & $30(54.5 \%)$ & $14(25.5 \%)$ & $14(25.5 \%)$ & $2(3.6 \%)$ & 0 \\
\hline
\end{tabular}

$\mathrm{ALT}=$ alanine aminotransferase, AST $=$ aspartate aminotransferase, CTCAE = National Cancer Institute Common Terminology Criteria for Adverse Events, INR = international normalized ratio

Table 3. Tumor Response to Chemoembolization

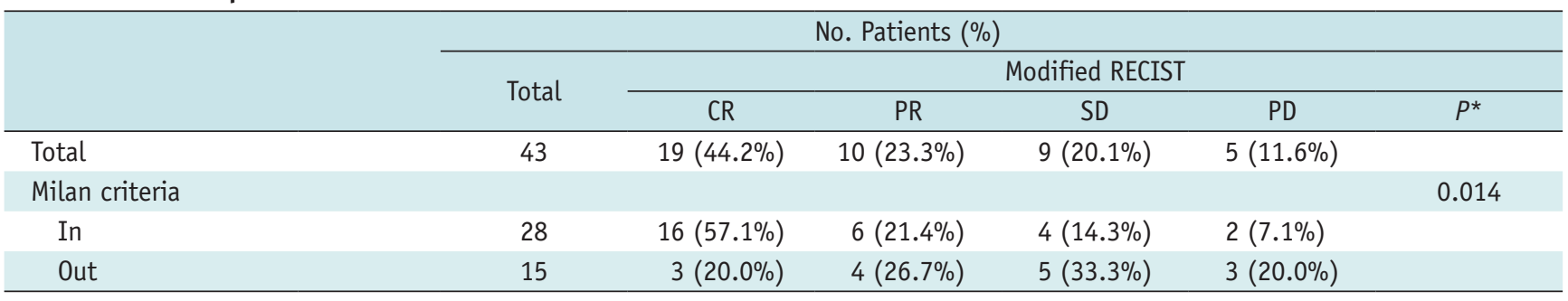

${ }^{*} P$ value was obtained using Mann-Whitney $\mathrm{U}$ test. $\mathrm{CR}=$ complete response, modified RECIST = modified Response Evaluation Criteria In Solid Tumors, $P D=$ progressive disease, $P R=$ partial response, $S D=$ stable disease 
patients underwent a liver transplantation at our institution after the chemoembolization. The median interval between their first chemoembolization and the transplantation was 5.1 months (range: 1.9-28 months). In six of these seven patients, the tumor burden before chemoembolization was within the Milan criteria, and the responses to chemoembolization were as follows: three complete responses, one partial response, one stable disease, and one progressive disease. The tumor burden of the remaining patient initially exceeded the Milan criteria, but the HCCs showed complete responses on follow-up imaging studies.

Among the remaining 48 patients who did not undergo liver transplantation, 15 patients underwent repeated chemoembolization and one underwent percutaneous ethanol injection therapy during the follow-up period.

\section{DISCUSSION}

Chemoembolization is commonly used for patients with unresectable HCC and has been shown to improve patients'

Table 4. Results of Univariate Analysis

\begin{tabular}{|c|c|c|c|c|c|c|c|}
\hline \multirow{2}{*}{ Factors } & & \multirow{2}{*}{$\begin{array}{l}\text { No. } \\
\text { Patients }\end{array}$} & \multirow{2}{*}{$\begin{array}{l}\text { Median Survival (Months) } \\
\qquad(95 \% \mathrm{CI})\end{array}$} & \multicolumn{4}{|c|}{ Overall Survival (\%) } \\
\hline & & & & 6 Month & $1 \mathrm{Yr}$ & $2 \mathrm{Yr}$ & $P^{*}$ \\
\hline \multirow{2}{*}{ Age } & $\leq 55$ & 29 & $8.8(2.4-15.3)$ & 57.8 & 31.1 & 17.8 & \multirow{2}{*}{0.816} \\
\hline & $>55$ & 26 & $6.9(4.0-9.9)$ & 57.4 & 30.9 & 17.7 & \\
\hline \multirow{2}{*}{ Sex } & Men & 46 & $6.9(3.1-10.8)$ & 51.3 & 25.6 & 15.4 & \multirow{2}{*}{0.183} \\
\hline & Women & 9 & $15.2(0-35.7)$ & 88.9 & 59.3 & 29.6 & \\
\hline \multirow{2}{*}{ ECOG PS } & $\leq 1$ & 24 & $12.9(4.3-21.5)$ & 70.4 & 50.3 & 35.2 & \multirow{2}{*}{0.003} \\
\hline & $>1$ & 31 & $4.0(0-8.2)$ & 47.1 & 15.7 & 3.9 & \\
\hline \multirow{2}{*}{ Child-Pugh score } & $\leq 10$ & 37 & $9.1(5.5-12.8)$ & 59.5 & 34.4 & 21.9 & \multirow{2}{*}{0.251} \\
\hline & $>10$ & 18 & $6.7(2.8-10.5)$ & 53.5 & 22.9 & 7.6 & \\
\hline \multirow{2}{*}{ MELD score } & $\leq 18$ & 36 & $9.1(5.5-12.8)$ & 61.0 & 37.3 & 23.7 & \multirow{2}{*}{0.109} \\
\hline & $>18$ & 19 & $6.7(1.1-12.3)$ & 50.4 & 18.9 & 6.3 & \\
\hline \multirow{2}{*}{ AJCC/UICC T staging } & 1 & 23 & $10.6(2.1-19.1)$ & 82.2 & 49.3 & 27.4 & \multirow{2}{*}{0.008} \\
\hline & $>1$ & 32 & $3.6(2.8-4.4)$ & 40.4 & 18.4 & 11.0 & \\
\hline \multirow{2}{*}{ CLIP score } & $\leq 3$ & 37 & $9.8(7.5-12.1)$ & 77.8 & 44.0 & 23.7 & \multirow{2}{*}{$<0.001$} \\
\hline & $>3$ & 18 & $2.4(0.8-4.0)$ & 16.7 & 5.6 & 5.6 & \\
\hline \multirow{2}{*}{ Okuda staging } & $\leq 2$ & 31 & $9.7(6.1-13.2)$ & 64.5 & 35.8 & 21.5 & \multirow{2}{*}{0.129} \\
\hline & 3 & 24 & $4.6(0.1-9.2)$ & 48.3 & 24.1 & 12.1 & \\
\hline \multirow{2}{*}{ Milan criteria } & In & 30 & $10.6(6.2-15.0)$ & 82.8 & 45.6 & 24.8 & \multirow{2}{*}{$<0.001$} \\
\hline & Out & 25 & $3.3(2.5-4.1)$ & 28.0 & 14.0 & 9.3 & \\
\hline \multirow{2}{*}{ Portal vein thrombosis } & Absent & 42 & $9.7(7.2-12.1)$ & 73.2 & 41.0 & 23.4 & \multirow{2}{*}{$<0.001$} \\
\hline & Present & 13 & $2.8(1.8-3.8)$ & 7.7 & 0.0 & 0.0 & \\
\hline \multirow{2}{*}{ Serum AFP (ng/mL) } & $\leq 400$ & 39 & $9.1(5.9-12.3)$ & 68.4 & 39.1 & 22.8 & \multirow{2}{*}{0.018} \\
\hline & $>400$ & 16 & $3.4(3.2-3.6)$ & 31.3 & 12.5 & 6.3 & \\
\hline \multirow{2}{*}{ Serum albumin $(\mathrm{g} / \mathrm{dL})$} & $\geq 2.5$ & 34 & $8.8(3.3-14.3)$ & 57.8 & 33.3 & 16.5 & \multirow{2}{*}{0.942} \\
\hline & $<2.5$ & 21 & $7.0(4.0-10.0)$ & 57.1 & 28.6 & 19.0 & \\
\hline Serum hiliruhin $(\mathrm{ma} / \mathrm{dl})$ & $\leq 3$ & 13 & $9.1(2.7-15.5)$ & 61.5 & 26.4 & 17.6 & $0<995$ \\
\hline (mitul) & $>3$ & 42 & $7.1(3.6-10.6)$ & 56.4 & 32.6 & 17.8 & 0.495 \\
\hline Serum AST (TH/I) & $\leq 80$ & 26 & $9.8(6.8-12.9)$ & 80.8 & 44.4 & 28.3 & 0004 \\
\hline 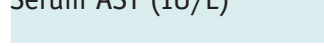 & $>80$ & 29 & $3.4(2.4-4.4)$ & 35.8 & 17.9 & 6.0 & 0.004 \\
\hline Sorum AIT (TI/I) & $\leq 40$ & 22 & $9.1(5.3-13.0)$ & 68.2 & 42.0 & 26.2 & 0154 \\
\hline 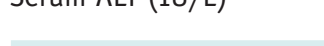 & $>40$ & 33 & $7.1(2.3-11.9)$ & 50.5 & 23.3 & 11.7 & 0.134 \\
\hline Tumer recnonse & CR/PR & 29 & $13.4(8.8-18.1)$ & 78.2 & 56.5 & 30.4 & $<0001$ \\
\hline 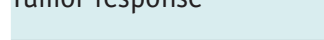 & $\mathrm{SD} / \mathrm{PD}^{\dagger}$ & 26 & $2.8(1.8-3.9)$ & 34.6 & 4.3 & 4.3 & -0.001 \\
\hline
\end{tabular}

* $P$ value was obtained using log-rank test, ${ }^{\dagger}$ Patients who had no follow-up image were considered as progressive disease. AJCC/ UICC = American Joint Committee on Cancer/Union for International Cancer Control, ALT = alanine aminotransferase, AST $=$ aspartate aminotransferase, $\mathrm{CI}=$ confidence interval, CLIP $=$ Cancer of the Liver Italian Program, CR $=$ complete response, ECOG $=$ Eastern Cooperative Oncology Group, INR = international normalized ratio, MELD = Model for End-Stage Liver Disease, $\mathrm{PD}=$ progressive disease, $\mathrm{PR}=$ partial response, $\mathrm{PS}=$ performance status, $\mathrm{SD}=$ stable disease 


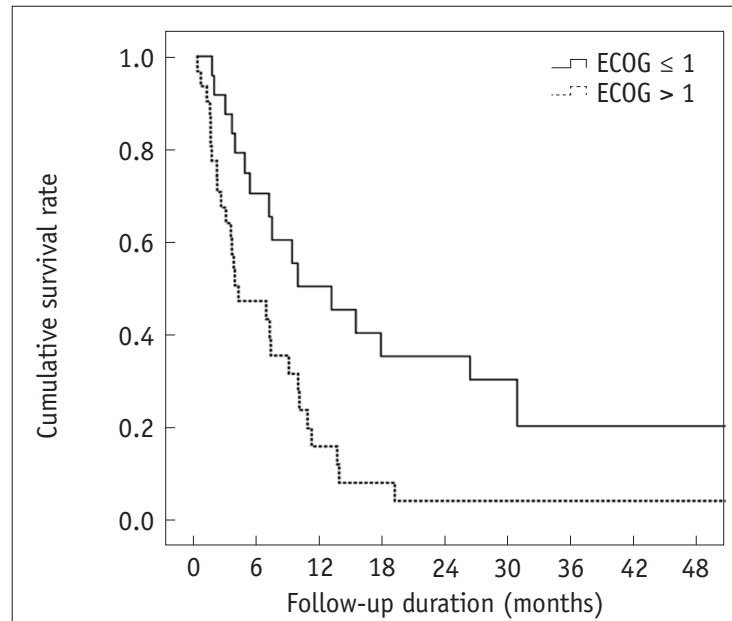

Number at risk

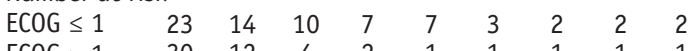

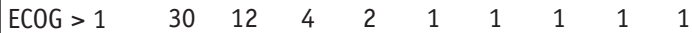

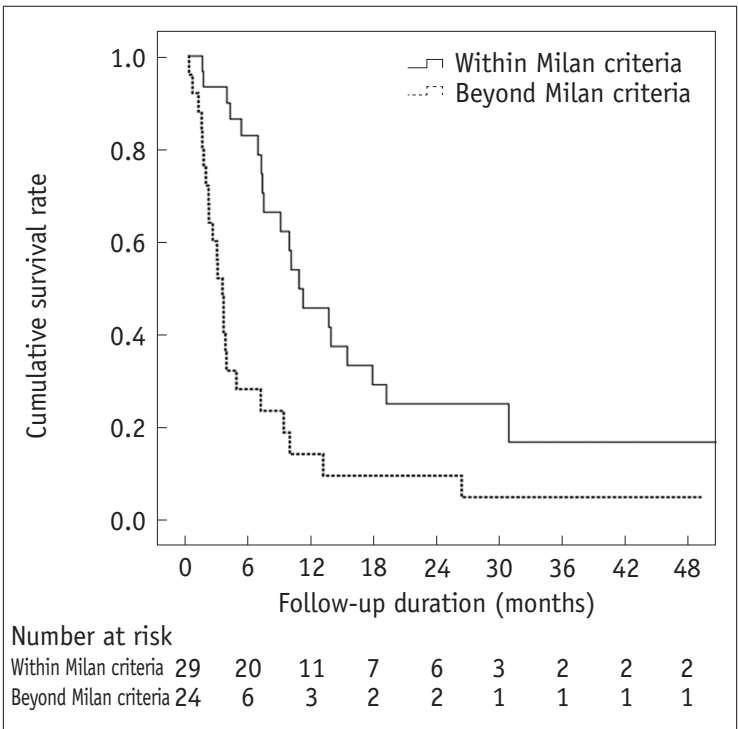

B

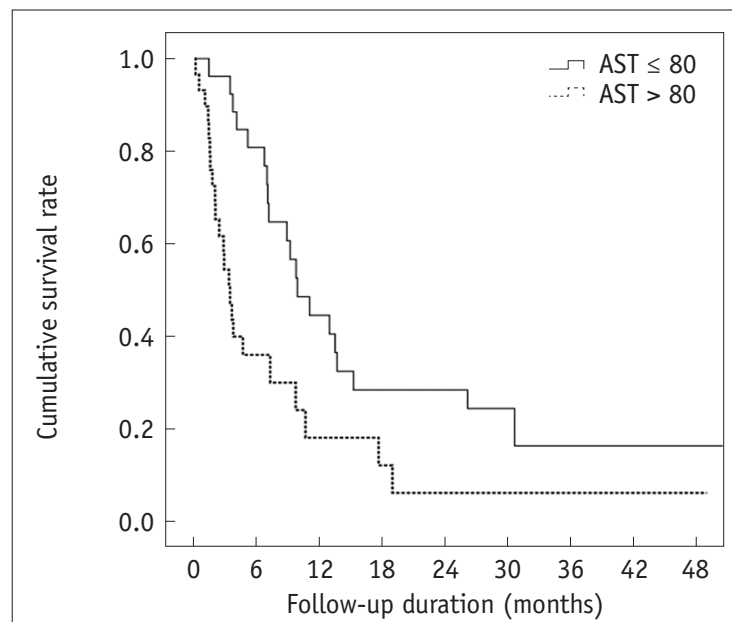

Number at risk

$\begin{array}{llllllllll}\text { AST } \leq 80 & 25 & 20 & 11 & 7 & 7 & 3 & 2 & 2 & 2 \\ \text { AST }>80 & 28 & 6 & 3 & 2 & 1 & 1 & 1 & 1 & 1\end{array}$

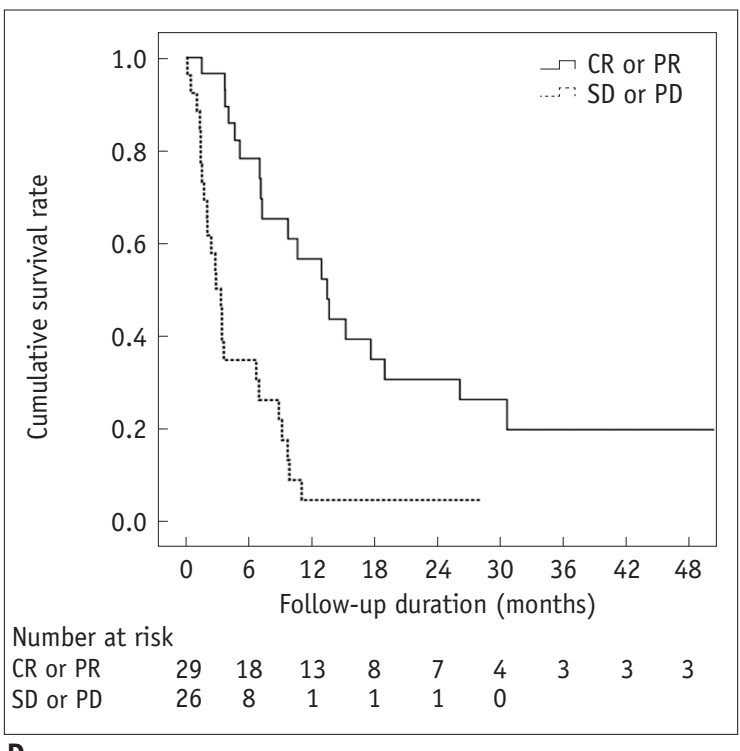

D

Fig. 2. Cumulative survival rates according to prognostic factors.

A. Survival curves of patients whose ECOG performance status was 1 vs. more than 1 (median survival time, 12.9 months; $95 \%$ CI, $4.3-21.5$ vs. 4.0 months; $95 \%$ CI $, 0-8.2, p=0.003$ ). B. Survival curves of patients whose tumor burden was within Milan criteria vs. beyond Milan criteria (median survival time, 10.6 months; $95 \%$ CI, $6.2-15.0$ vs. 3.3 months; $95 \%$ CI, 2.5-4.1, $p<0.001$ ). C. Survival curves of patients with serum aspartate aminotransferase levels $\leq 80 \mathrm{IU} / \mathrm{L}$ vs. $>80 \mathrm{IU} / \mathrm{L}$ (median survival time, 9.8 months; $95 \%$ CI, 6.8-12.9 vs. 3.4 months; $95 \%$ CI, $2.4-4.4$, $p=0.004)$. D. Survival curves of patients with presence of tumor response (complete or partial response) vs. absence of tumor response (stable or progressive disease) (median survival time, 13.4 months; $95 \%$ CI, 8.8-18.1 vs. 2.8 months; 95\% CI, 1.8-3.9, $p<0.001$ ). AST = aspartate aminotransferase, $\mathrm{CI}=$ confidence interval, $\mathrm{CR}=$ complete response, $\mathrm{ECOG}=$ Eastern Cooperative Oncology Group, $\mathrm{PD}=$ progressive disease, $\mathrm{PR}=$ partial response, $\mathrm{SD}=$ stable disease

survival compared with best supportive care (3-5). Although a consensus has not yet been reached, the best candidates for chemoembolization appear to be asymptomatic patients with preserved liver function and without vascular invasion or extrahepatic tumor metastasis (5). In fact, the benefits of chemoembolization should not be offset by treatmentinduced liver damage. Thus, to minimize the injury to normal liver parenchyma, chemoembolization should be performed by selective catheterization of the hepatic segmental or subsegmental arteries feeding the tumor.

In this study, the overall incidence of chemoembolizationrelated major complications in Child-Pugh class $\mathrm{C}$ patients was $9.1 \%$, which is within the acceptable threshold (15\%) suggested by the Quality Improvement Guidelines for 
Table 5. Results of Multivariate Analysis

\begin{tabular}{lccc}
\hline & Hazard Ratio & $95 \%$ CI & $P$ \\
\hline ECOG Performance Status $>1$ & 2.924 & $1.399-6.108$ & 0.004 \\
Beyond Milan criteria & 2.956 & $1.231-7.102$ & 0.015 \\
Portal vein thrombosis & 1.432 & $0.494-4.153$ & 0.509 \\
Serum AFP level $>400(\mathrm{ng} / \mathrm{mL})$ & 0.846 & $0.421-1.699$ & 0.638 \\
Serum AST level $>80(\mathrm{IU} / \mathrm{L})$ & 2.532 & $1.254-5.112$ & 0.010 \\
Stable or progressive disease & 4.247 & $1.925-9.369$ & $<0.001$ \\
\hline
\end{tabular}

$\mathrm{AFP}=$ alpha-fetoprotein, AST = aspartate aminotransferase, $\mathrm{CI}=$ confidence interval, ECOG = Eastern Cooperative Oncology Group, INR = international normalized ratio

Transhepatic Arterial Chemoembolization, Embolization, and Chemotherapeutic Infusion for Hepatic Malignancy of the Society of Interventional Radiology (14). The results of our study also showed that the patients with major complications were more likely to be those whose tumor burden exceeded the Milan criteria $(p=0.015)$ and who had significantly higher CLIP scores $(p=0.003)$ compared with those without complications or with minor complications. Consequently, each patient's tumor burden appeared to be closely related to the development of major complications after chemoembolization. The results of our study are in good agreement with those of previous studies of high-risk patient groups, although a large portion of the subjects in those studies were Child-Pugh class B patients $(15,16)$.

According to BCLC guidelines, if a liver transplantation is not indicated, there are no available treatment options, including surgical resection, radiofrequency ablation, or chemoembolization, for Child-Pugh class C patients because of their poor natural clinical courses caused by severely compromised hepatic function and concerns about treatment-related toxicity (1). However, there has been a wide discrepancy between the number of available donor organs and the waiting list of transplantation candidates, which has consequently led to long waiting periods for transplantation. In this context, a previous study by Dhanasekaran et al. (17) demonstrated a role for chemoembolization as a "bridging therapy" to control and maintain tumor burden within the Milan criteria during that waiting period in Child-Pugh class A and B candidates. In addition, our study also showed that the patients whose tumor burden was within the Milan criteria had significantly lower and relatively acceptable incidence of major chemoembolization-related complications and a higher tumor response rate to chemoembolization compared with those with a large tumor burden. Therefore, we suggest that even in patients with decreased liver function classified as Child-Pugh class C, in selected cases with small tumor burden within the Milan criteria, chemoembolization can be considered and performed safely as a bridging therapy before transplantation. Among the subjects in this study, seven later underwent a liver transplantation after chemoembolization.

We speculate that this selective technique, which was used in all technically feasible cases, may have played a key role in minimizing non-tumorous liver parenchymal damage and consequent hepatic function deterioration. Although a previous study by Caturelli et al. (18) showed that chemoembolization performed in proper or main hepatic arteries does not induce significant long-term deterioration of hepatic function in patients with Child-Pugh class $A$ and $B$ cirrhosis, this result cannot be consistently applied to Child-Pugh class $C$ patients whose liver function is severely compromised. Reports have shown the effects of chemoembolization on non-tumorous liver parenchyma that led to immediate worsening of liver function $(19,20)$. Furthermore, Miyayama et al. (21) evaluated histopathological findings after chemoembolization and demonstrated that chemoembolization, if properly performed, induces peritumoral parenchymal necrosis as well as complete tumor necrosis. Therefore, to lower the incidence of procedure-related complications, it is essential to catheterize the most distal branches of the hepatic artery with a microcatheter to minimize the embolized area. Recently, there have been great advances in flat-panel detector and cone-beam CT technology, which are useful for detecting distal branches of hepatic arteries and small tumor-feeding arteries (22-24). Therefore, the discrepancy between the results of our study and those of the previous literature that showed an extremely high incidence of chemoembolization-related morbidity and mortality in patients with Child-Pugh class C cirrhosis may partly be explained by the differences in chemoembolization methods and techniques (25). Recently, Kothary et al. (15) reported the safety and clinical outcomes of chemoembolization 
in a high-risk patient group, including 14 Child-Pugh class C patients (14/52, 26.9\%). They performed selective chemoembolization in all technically feasible cases $(56.9 \%$ of all procedures) and reported that 30-day mortality was $7.7 \%$ and that the morbidity rate was $10.8 \%$, which are similar to the results of our study. In addition, they reported that the mortality of patients who underwent lobar chemoembolization was significantly higher than that of patients who were treated with the selective technique.

Our study has a number of limitations. First, because this was a retrospective study with a relatively small number of patients, there may have been a selection bias. Second, the intervals between the chemoembolization procedure and follow-up laboratory tests, as well as the imaging studies, could not be controlled uniformly owing to the study's retrospective nature. Third, this study did not have a matched control group, and we compared and discussed the results of our study with those of the previous literature. Therefore, additional matched randomized studies with a large study population will be required to confirm our results and speculations.

In conclusion, even in patients with decreased liver function classified as Child-Pugh class C, chemoembolization can be performed safely in selected cases with a small tumor burden.

\section{REFERENCES}

1. Forner A, Reig ME, de Lope CR, Bruix J. Current strategy for staging and treatment: the BCLC update and future prospects. Semin Liver Dis 2010;30:61-74

2. Hwang S, Lee SG, Belghiti J. Liver transplantation for HCC: its role: Eastern and Western perspectives. J Hepatobiliary Pancreat Sci 2010;17:443-448

3. Llovet JM, Real MI, Montaña X, Planas R, Coll S, Aponte J, et al. Arterial embolisation or chemoembolisation versus symptomatic treatment in patients with unresectable hepatocellular carcinoma: a randomised controlled trial. Lancet 2002;359:1734-1739

4. Llovet JM, Bruix J. Systematic review of randomized trials for unresectable hepatocellular carcinoma: chemoembolization improves survival. Hepatology 2003;37:429-442

5. Bruix J, Sala M, Llovet JM. Chemoembolization for hepatocellular carcinoma. Gastroenterology 2004;127(5 Suppl 1):S179-S188

6. Eiber M, Holzapfel K, Ganter C, Epple K, Metz S, Geinitz H, et al. Whole-body MRI including diffusion-weighted imaging (DWI) for patients with recurring prostate cancer: technical feasibility and assessment of lesion conspicuity in DWI. J

Magn Reson Imaging 2011;33:1160-1170
7. Miyayama S, Matsui 0, Yamashiro M, Ryu Y, Kaito K, Ozaki K, et al. Ultraselective transcatheter arterial chemoembolization with a 2-f tip microcatheter for small hepatocellular carcinomas: relationship between local tumor recurrence and visualization of the portal vein with iodized oil. J Vasc Interv Radiol 2007;18:365-376

8. Kim HC, Chung JW, Jae HJ, Yoon JH, Lee JH, Kim YJ, et al. Caudate lobe hepatocellular carcinoma treated with selective chemoembolization. Radiology 2010;257:278-287

9. Bruix J, Sherman M; American Association for the Study of Liver Diseases. Management of hepatocellular carcinoma: an update. Hepatology 2011;53:1020-1022

10. Mazzaferro V, Regalia E, Doci R, Andreola S, Pulvirenti A, Bozzetti $F$, et al. Liver transplantation for the treatment of small hepatocellular carcinomas in patients with cirrhosis. $N$ Engl J Med 1996;334:693-699

11. National Cancer Institute. Common terminology criteria for adverse events (CTCAE). version 4.03. http://evs.nci.nih. gov/ftp1/CTCAE/. Accessed June 14, 2010

12. Leung DA, Goin JE, Sickles C, Raskay BJ, Soulen MC. Determinants of postembolization syndrome after hepatic chemoembolization. J Vasc Interv Radiol 2001;12:321-326

13. Lencioni R, Llovet JM. Modified RECIST (mRECIST) assessment for hepatocellular carcinoma. Semin Liver Dis 2010;30:52-60

14. Brown DB, Nikolic B, Covey AM, Nutting CW, Saad WE, Salem R, et al. Quality improvement guidelines for transhepatic arterial chemoembolization, embolization, and chemotherapeutic infusion for hepatic malignancy. J Vasc Interv Radiol 2012;23:287-294

15. Kothary N, Weintraub JL, Susman J, Rundback JH. Transarterial chemoembolization for primary hepatocellular carcinoma in patients at high risk. J Vasc Interv Radiol 2007;18:1517-1526; quiz 1527

16. Kiely JM, Rilling WS, Touzios JG, Hieb RA, Franco J, Saeian $\mathrm{K}$, et al. Chemoembolization in patients at high risk: results and complications. J Vasc Interv Radiol 2006;17:47-53

17. Dhanasekaran R, Khanna V, Kooby DA, Spivey JR, Parekh $S$, Knechtle SJ, et al. The effectiveness of locoregional therapies versus supportive care in maintaining survival within the Milan criteria in patients with hepatocellular carcinoma. J Vasc Interv Radiol 2010;21:1197-1204; quiz 1204

18. Caturelli E, Siena DA, Fusilli S, Villani MR, Schiavone G, Nardella M, et al. Transcatheter arterial chemoembolization for hepatocellular carcinoma in patients with cirrhosis: evaluation of damage to nontumorous liver tissue-long-term prospective study. Radiology 2000;215:123-128

19. Miyoshi S, Minami Y, Kawata S, Imai Y, Saitoh R, Noda S, et al. Changes in hepatic functional reserve after transcatheter embolization of hepatocellular carcinoma. Assessment by maximal removal rate of indocyanine green. $J$ Hepatol 1988;6:332-336

20. Khan KN, Nakata K, Kusumoto Y, Shima M, Ishii N, Koji $T$, et al. Evaluation of nontumorous tissue damage by 
transcatheter arterial embolization for hepatocellular carcinoma. Cancer Res 1991;51:5667-5671

21. Miyayama S, Mitsui T, Zen Y, Sudo Y, Yamashiro M, Okuda $M$, et al. Histopathological findings after ultraselective transcatheter arterial chemoembolization for hepatocellular carcinoma. Hepatol Res 2009;39:374-381

22. Miyayama S, Yamashiro M, Okuda M, Yoshie Y, Sugimori $N$, Igarashi S, et al. Usefulness of cone-beam computed tomography during ultraselective transcatheter arterial chemoembolization for small hepatocellular carcinomas that cannot be demonstrated on angiography. Cardiovasc Intervent Radiol 2009;32:255-264

23. Choi WS, Kim HC, Hur S, Choi JW, Lee JH, Yu SJ, et al.
Role of C-arm CT in identifying caudate arteries supplying hepatocellular carcinoma. J Vasc Interv Radiol 2014;25:13801388

24. Lee IJ, Chung JW, Yin YH, Kim HC, Kim YI, Jae HJ, et al. Cone-beam CT hepatic arteriography in chemoembolization for hepatocellular carcinoma: angiographic image quality and its determining factors. J Vasc Interv Radiol 2014;25:13691379; quiz 1379-1379.e1

25. Bismuth H, Morino M, Sherlock D, Castaing D, Miglietta C, Cauquil P, et al. Primary treatment of hepatocellular carcinoma by arterial chemoembolization. Am J Surg 1992;163:387-394 